\title{
Comportement en service des principaux ouvrageses de génie civil de l'usine de la Rance
}

\author{
par L. Duhoux \\ Ingénieur E.D.F., \\ Ancien Chef de la Division Génie civil \\ de l'Aménagement de la Rance.
}

C'est parce que le comportement des ouvrages de génie civil de la Rance a été excellent que cette communication ne donne pas lieu à de longs développements et à des analyses savantes.

On dit, en effet, que «les gens heureux n'ont pas d'histoire » et il en est de même, sans doute, pour les ouvrages solides.

Pourtant, il est nécessaire, après six années d'exploitation $\left(^{a}\right)$, de faire le point de la tenue de ce grand ouvrage en béton armé, qui est à la fois barrage et usine sousmarine, soumis à un travail mécanique constant et varié sous l'influence de la marée et qui est soumis aussi à une action continue et toujours dangereuse de corrosion par l'eau de mer.

On a, évidemment, construit beaucoup d'ouvrages maritimes en béton armé ou précontraint dans le monde avant l'usine de la Rance et on en construit encore beaucoup maintenant, mais peu ont cette importance et cette complexité de formes.

Avant d'examiner le comportement de l'ouvrage dans différents domaines, je pense qu'il est utile de rappeler quelques-unes de ses caractéristiques et les conditions dans lesquelles il a été étudié et exécuté.

\section{Caractéristiques.}

L'ensemble de l'aménagement comprend d'ouest en est:

- une écluse de $13 \mathrm{~m}$ de largeur, avec un radier à $(+2,00)$ et des bajoyers constitués par des murs-poids;

( $\left.{ }^{a}\right)$ L'écluse a été mise en service en novembre 1962 et les pertuis en janvier 1963. L'usine a été mise en eau en mars 1966.
- l'usine proprement dite, de $380 \mathrm{~m}$ de longueur, de $53,5 \mathrm{~m}$ de largeur à la base et de 31 à $33 \mathrm{~m}$ de hauteur comprenant :

- des locaux accolés à l'écluse,

- 3 travées de montage,

- 1 travée de commande,

- 24 travées de groupe;

- une digue de $150 \mathrm{~m}$ de longueur en enrochements, avec noyau central en béton;

- 6 pertuis de vannage, équipés de 6 vannes de $15 \times 10 \mathrm{~m}$, s'appuyant à la partie inférieure sur des radiers à $(-4,00)$.

Cet ensemble a nécessité la mise en œuvre ou l'exécution de :

- $350000 \mathrm{~m}^{3}$ de béton;

- $16000 \mathrm{t}$ d'acier $\left(65 \mathrm{~kg} / \mathrm{m}^{3}\right.$ en infrastructure de [-14,00] à $[0,00]$ et $120 \mathrm{~kg} / \mathrm{m}^{3}$ en superstructure);

- $275000 \mathrm{~m}^{2}$ de coffrages.

La surface totale des parements exposés à l'eau de mer est d'environ $90000 \mathrm{~m}^{2}$.

\section{Conception de l'usine.}

Du point de vue stabilité, l'ouvrage résiste par son poids aux actions suivantes :

a) Charge d'eau, avec un niveau côté mer oscillant entre 0,00 et 14,50 et un niveau côté bassin oscillant entre 0,00 et 13,50 (voir fig. 1 ).

b) Sous-pression partielle.

c) Séisme, donnant une accélération de $\mathrm{g} / 20$, ce qui correspond à une intensité 6 à l'échelle Mercali; $g$ étant l'accélération gravitaire. 


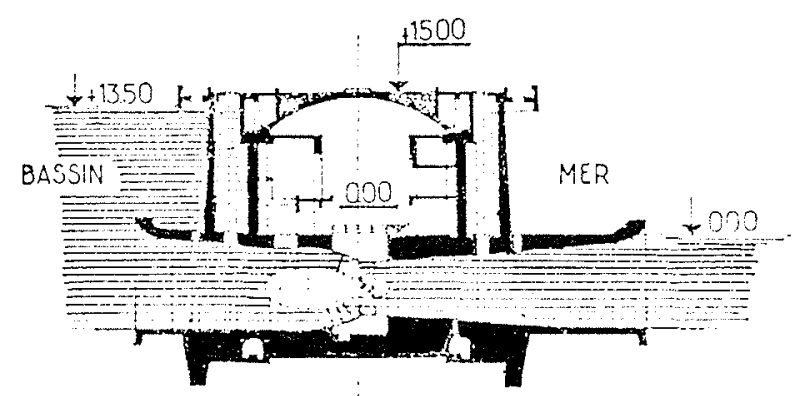

béton. Il en est par conséquent résulté un alourdissement des ouvrages.

Les éléments sont dimensionnés de façon que les contraintes de traction dans le béton, sur les parements exposés à l'eau de mer, ne dépassent pas 25 bars en considérant le matériau homogène et sans armatures. En complément, la contrainte maxima admise dans les aciers pour ces sections, si elles fissurent malgré cette première condition concernant le béton, est de 10 hectobars (fig. 2).

Ces conditions judicieuses ont d'ailleurs été reprises, mieux explicitées et complétées par les règlements officiels sur le béton armé et, notamment, par celui de 1968. On tient compte, à juste titre, du diamètre des barres, d'un coefficient de fissuration propre au type d'armature et d'un second coefficient propre aux conséquences de la fissuration et qui dépend de la largeur des fissures.

Pour les grands ouvrages maritimes, cela conduit à une augmentation des prix de revient et aussi, souvent, à des surdimensionnements.

Par exemple, pour la grande écluse du port du Havre, qui vient d'être mise en service, dont le radier a $4,50 \mathrm{~m}$ de hauteur et qui a nécessité la mise en cuvre de $37000 \mathrm{t}$ d'acier dans $470000 \mathrm{~m}^{3}$ de béton, on n'a pas utilisé d'armatures d'un diamètre supérieur à $25 \mathrm{~mm}$.

\section{Exécution.}

Il semble également utile d'ajouter quelques renseignements concernant l'exécution.

Tous les ouvrages ont été exécutés à sec dans des enceintes batardées, c'est-à-dire dans de bonnes conditions. Souvent, en effet, en technique de travaux maritimes, on coule du béton «à la marée» ou sous l'eau et la qualité des ouvrages s'en ressent inévitablement.

Les travaux ont été réalisés par l'entreprise Tramarance,

1/ Principaux cas de charges.

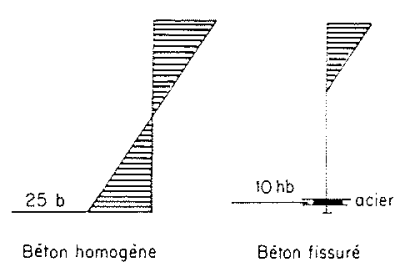

2/ Contraintes dans le béton armé.

Sous ces actions, la stabilité au renversement est assurée avec un coefficient de sous-pression supérieur à 0,75 sans tenir compte des séismes et de 0,60 avec effet séismique. Pour la stabilité au glissement, le rapport de la résultante des forces horizontales et de la résultante des forces verticales, s'exerçant sur l'assise, n'excède pas 0,6 sans effet séismique et n'excède pas 0,75 avec effet séismique.

Enfin, en supposant une répartition linéaire de contraintes sur la fondation, le taux de travail du rocher est de $4 \mathrm{~kg} / \mathrm{cm}^{2}$ ce qui est très faible compte tenu de sa qualité.

Dans le calcul des structures de l'ouvrage soumises au marnage, on a considéré qu'il fallait limiter au minimum le phénomène de fissuration et, dans ce but, on a réduit la valeur des contraintes admissibles dans l'acier et dans le groupement issu des entreprises suivantes:

— Société Générale d'Entreprises;

- Fougerolle;

- Campenon Bernard;

- Entreprise de Grands Travaux Hydrauliques;

- Société Française d'Entreprises de Dragages et de Travaux Publics.

Il est juste de lui rendre hommage pour le parfait établissement de tous les plans détaillés d'exécution, pour la bonne organisation de son chantier et pour les soins qu'elle a apportés à la mise en œuvre.

Cette qualité d'exécution est, en effet, absolument primordiale pour la bonne tenue des ouvrages maritimes. Une structure en béton armé, judicieusement calculée et devant être réalisée avec un excellent béton étudié en laboratoire, sera finalement rapidement menacée si les ouvriers et cadres de l'entreprise ne veillent pas, dans le détail, à la position des armatures, à la distribution du béton, à sa vibration et à sa conservation.

Cela pose beaucoup de problèmes quand on travaille, par exemple, une nuit d'hiver, sous la pluie, à la fin d'un poste harassant de huit ou dix heures!

Le délai total d'exécution a été de six ans environ, mais l'exécution de l'usine proprement dite, représentant $250000 \mathrm{~m}^{3}$ de béton, $210000 \mathrm{~m}^{2}$ de coffrage et $14000 \mathrm{t}$ d'acier n'a été que de 28 mois $\left(^{b}\right)$ (le volume maximum de béton mis en ceuvre par mois ayant été de $16000 \mathrm{~m}^{3}$ ).

(") Du $1^{\text {er }}$ décembre 1963 à mars 1966 
De son côté, E.D.F. a conçu et projeté l'ensemble des ouvrages et en a assuré le contrôle. Dans le laboratoire du chantier, il a effectué de nombreuses études avant et pendant l'exécution. Elles ont porté notamment sur le ciment, la composition du béton et le comportement des armatures. L'installation d'un laboratoire de chantier bien équipé a été un élément important du contrôle effectué par les agents E.D.F.

Les chiffres suivants indiquent l'importance de ce contrôle de qualité :

- 12000 éprouvettes de ciment;

- 250 analyses granulométriques;

- 5600 éprouvettes de béton;

- 250 carottages dans l'ouvrage.

Ces chiffres ne tiennent pas compte des études particulières (soixante-dix environ).

Pour assurer la pérennité des ouvrages, les dispositions suivantes ont été - entre autres - prises :

- très bon traitement du rocher;

- utilisation d'un ciment de haut-fourneau, en provenance de Colombelles, contenant $75 \%$ de laitier, difficilement attaquable par l'eau de mer, ayant une grande finesse et une faible chaleur d'hydratation;

- lavage et coupure des sables de mer du littoral en deux catégories, de façon à avoir un composé assez régulier; refus d'utiliser du sable de Rance;

- utilisation d'un agrégat de qualité, en provenance de Pleherel, situé à $30 \mathrm{~km}$ du chantier, et lavage des matériaux;

- composition d'un béton riche en ciment (350 ou $400 \mathrm{~kg}$ ) et assez pauvre en sable;

- utilisation d'un plastifiant réducteur d'eau;

- pervibration aussi soignée que possible;

- armatures en provenance de la même usine;

- distance des armatures aux parements au moins égale à $5 \mathrm{~cm}$.

Evidemment, tout n'a pas été parfait. Parce qu'il y a des prix de revient, et que les hommes et les machines ont des défaillances ou commettent des erreurs, un visiteur attentif trouvera de petits défauts dans l'ouvrage et un entretien est toujours indispensable.

Mais portons maintenant notre analyse sur les points suivants qui nous paraissent essentiels :

$1^{\circ}$ L'étanchéité générale de l'usine.

$2^{\circ}$ La tenue des structures en béton armé.

$3^{\circ}$ Le tassement de la digue.

$4^{0}$ Les affouillements.

\section{1. - Etanchéité générale de l'usine}

C'était, évidemment, une condition indispensable, pour l'exploitation, que l'usine soit étanche afin de garantir le fonctionnement et le facile entretien des matériels mécaniques et électriques.

En entrant au niveau $(0,00)$, on est frappé par l'aspect impeccable de tous les parements. Depuis la voûte jusqu'au fond des fosses du groupe, on n'aperçoit aucune tâche d'humidité, même quand on va derrière les masques regarder de plus près les parements verticaux soumis à la charge d'eau.
La situation est presque aussi satisfaisante quand on descend dans les galeries à $(-12,00)$. Pourtant, la pression de l'eau peut atteindre 2,5 bars et l'entrepreneur a dû effectuer, lors de la construction, de très nombreuses reprises horizontales et verticales ou couler des bétons secondaires un peu partout. Il y a, d'autre part, $1,500 \mathrm{~km}$ de galeries et l'ouvrage a été fissuré dans la période de construction (nous reviendrons plus tard sur ce point).

Donc on peut dire que l'étanchéité est très satisfaisante et il est très difficile de préciser l'importance des quelques fuites en raison de leur faible valeur.

Elle est de l'ordre de 20 litres par minute $\left({ }^{c}\right)$, dont 10 litres proviennent de drains verticaux, réservés lors de la construction dans l'assise du mur de la digue et dans une zone d'usine traversée par une faille.

Le service de la Production n'a eu à effectuer que des travaux peu importants, consistant surtout en compléments d'injections pendant les hivers de 1968 à 1972, dont le coût moyen n'atteignait pas $40000 \mathrm{~F}$ par an et va en diminuant chaque année.

Cette excellente étanchéité est due à une conception judicieuse des ouvrages, à une bonne exécution du béton, mais aussi aux traitements importants réalisés, avant la mise en service, sur les joints, les fissures et les parements.

Rappelons l'importance de ces traitements complémentaires $\left({ }^{d}\right)$ :

1. Traitement de $5000 \mathrm{ml}$ de fissures, dont $4600 \mathrm{ml}$ par injection de produits souples à base de bitume, caoutchouc et résines synthétiques. Ces fissures étaient pour la grande majorité dues au retrait et notamment au retrait thermique.

2. Traitement, par colmatage au mastic, de $5100 \mathrm{ml}$ de reprises horizontales. Ce traitement a été facilité par la réservation d'une rainure lors du coulage.

$3^{\circ}$ Traitement, par colmatage au mastic, de $400 \mathrm{ml}$ de joints verticaux et notamment de ceux entre travées de montage ne comportant pas de joint en profilé Waterstop.

40 Exécution d'un revêtement général aux résines époxy, comportant un resurfaçage au mortier de résine sur $3200 \mathrm{~m}^{2}$ (travée 'de commande, travée de montage).

5. Exécution d'un traitement de parement destiné à rendre le béton non hygroscopique sur $9215 \mathrm{~m}^{2}$ (appliqué surtout sur les voiles entre $(0,00)$ et $(14,00)$.

Ces travaux ont entraîné une dépense de $2300000 \mathrm{~F}$ ce qui montre leur importance. Ils ont été précédés de nombreux essais en laboratoire.

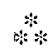

On constate que les parements ont été envahis par les balanes (ennemi numéro 1 des revêtements d'étanchéité) qui se développent particulièrement bien en Rance, mais ces animaux n'ont pas perforé les revêtements.

On doit, toutefois, noter des décollements et arrachements dans la partie supérieure des parements, tant du côté bassin que du côté mer. Cela montre que le revêtement ne doit plus durer très longtemps dans certaines zones (le délai de garantie est expiré et, d'autre part, les réparations sont difficiles à exécuter).

(c) Pour l'usine proprement dite (travées de groupe, travées de montage, travées de commande) et pour la digue.

( $\left.{ }^{l}\right)$ Exécutés par les Entreprises Terastic, Auboux et S.A.P.S. 


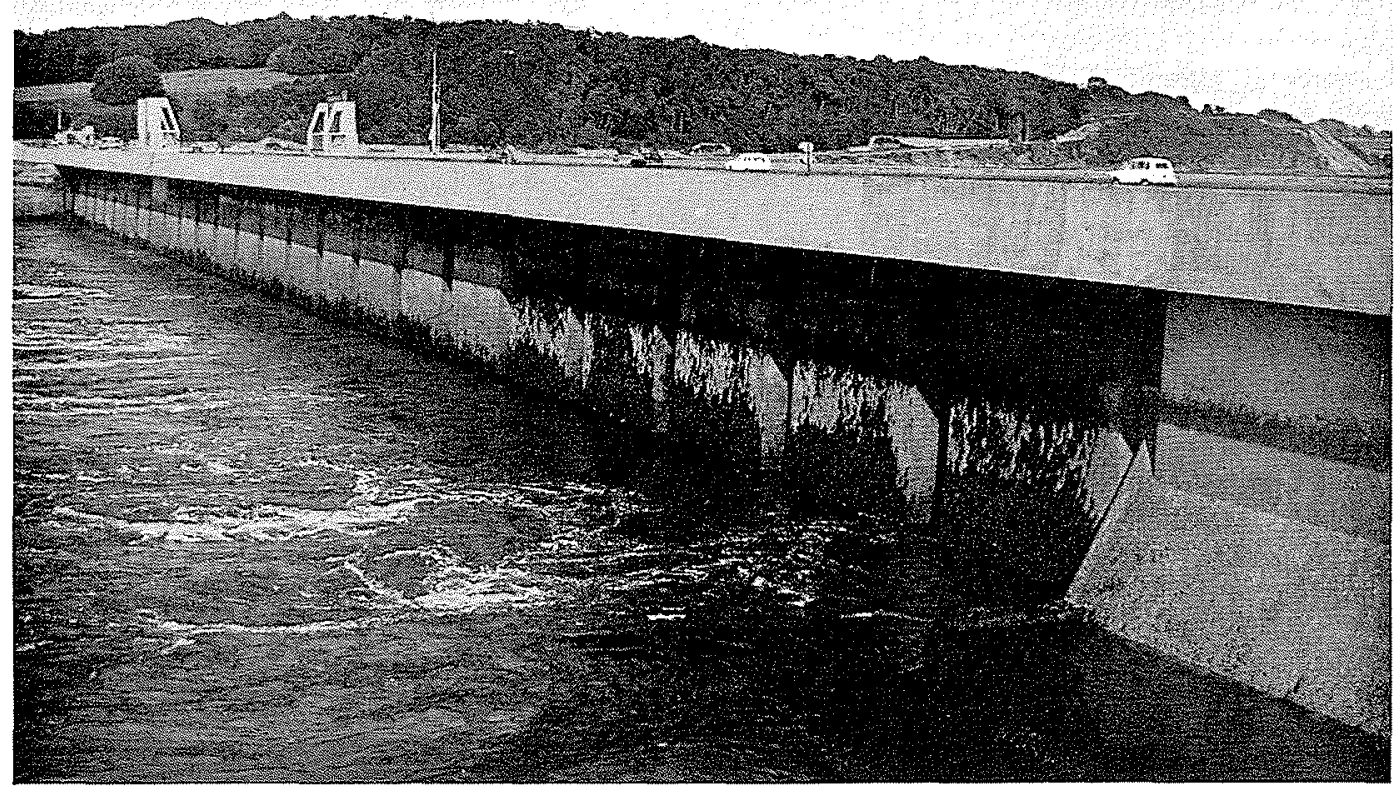

3/ Vue du parement côté mer.

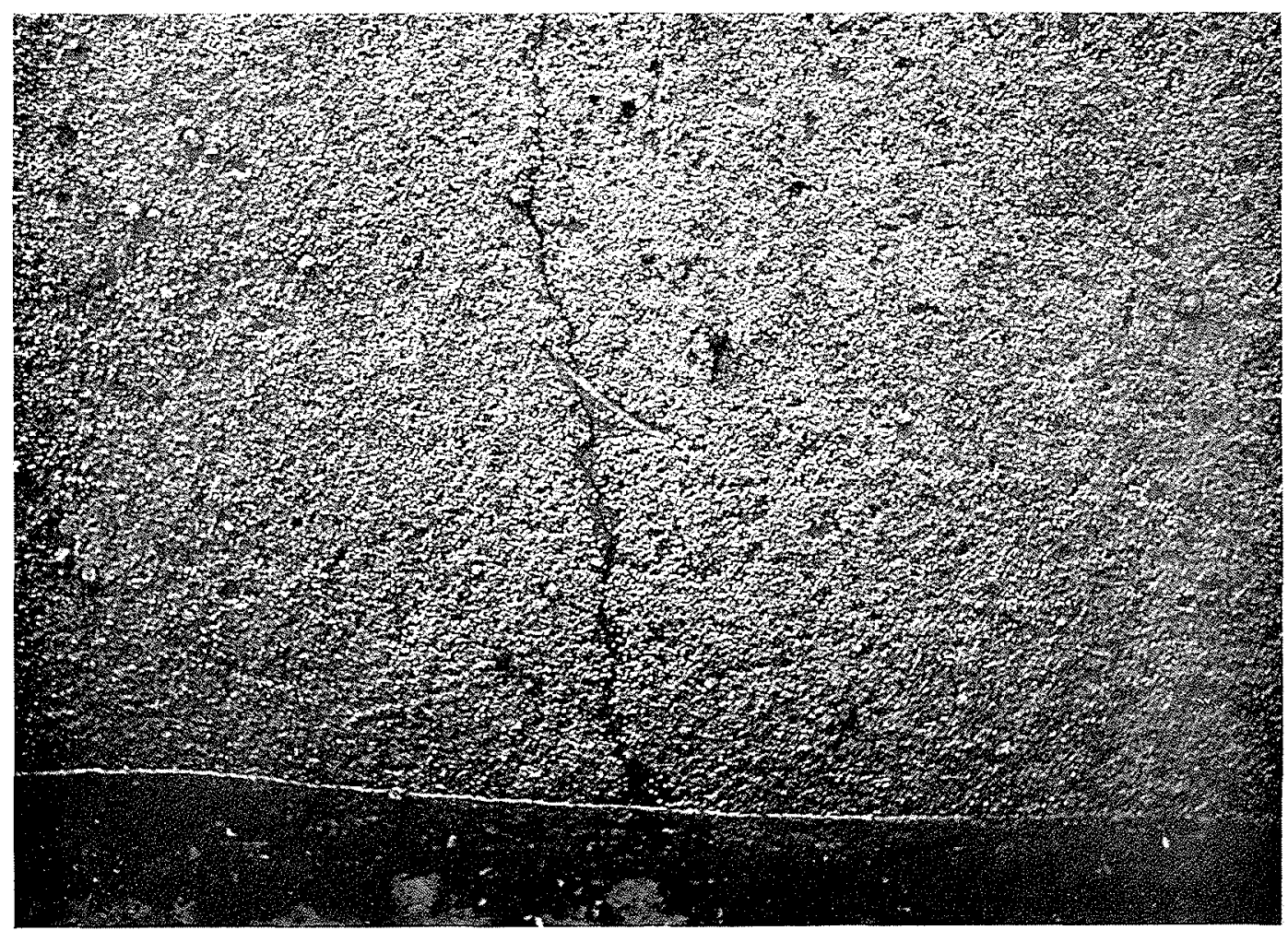

4/ Fissure non colmatée et non évolutive. 


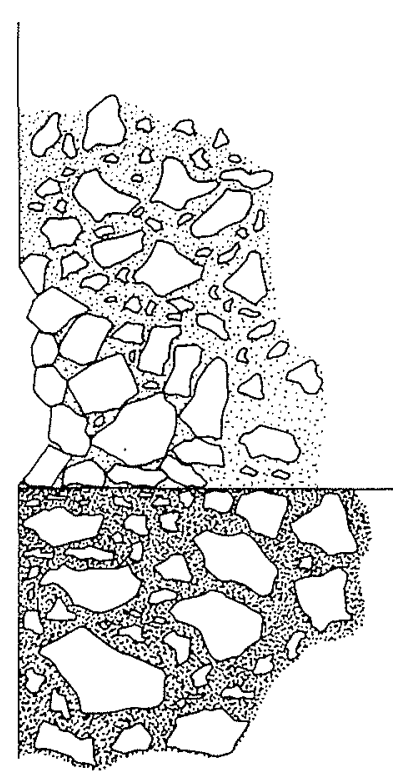

5/ Reprise de bétonnage.

\section{2. - Tenue de structures en béton armé}

Tous les ouvrages de la Rance se sont bien comportés pendant cette période de six années d'exploitation.

En les examinant, on ne relève pas de fissuration nouvelle importante ni de déformation anormale, ni de décomposition de béton, ni de corrosion dangereuse des armatures. L'ensemble est sain et doit durer longtemps.

Signalons, toutefois, les points suivants :

1. Le béton est recouvert, dans la zone immergée, par une épaisse couche de balanes qui atteint plusieurs centimètres et par des algues (fig. 3).

2. Les fissures de très faible ouverture se sont, pour la plupart, colmatées par formation de calcite.

Par contre, les fissures assez larges, $e>0,3 \mathrm{~mm}$, sont restées ouvertes et il me semble que certaines, comme celles des voiles des pertuis côté bassin (P. 5 notamment), seraient à traiter (fig. 4).

3. Les fissures importantes des radiers des pertuis de vannage, survenus par retrait thermique, ne semblent pas avoir évolué. Pourtant, le mortier de calfeutrement, à l'oxydo-ciment, a disparu en certains endroits et le produit souple Terastic apparaît.

Le calfeutrement serait donc à refaire, compte tenu de la grande vitesse d'écoulement $(10 \mathrm{~m} / \mathrm{seconde})$.

4. Plus de six années de séjour en eau de mer ont mis en évidence les petits défauts d'exécution cachés, volontairement ou non, lors de la mise en service.

Des colmatages n'ont pas tenu et les mauvaises reprises font apparaître des zones de béton poreux (fig. 5).

5. On note, également, un assez grand appauvrissement en fines de certaines zones du parement. Une mosaïque da graviers apparaît de la même façon que lors d'un sablage (poutres du pont sur pertuis, partie nord par exemple).

On peut se demander si la fragilité de cette laitance superficielle n'est pas due à l'emploi du ciment de laitier qui a pu faire une mauvaise prise, car il n'a pas durci dans des conditions idéales d'humidité. On a constaté ce phénomène sur d'autres chantiers.

C'est un point à suivre (fig. 6).

Là encore, on peut penser qu'il eût peut-être mieux valu couler les superstructures, au-dessus du niveau moyen ou supérieur des marées, en ciment Portland, en n'utilisant le C.H.F. que pour les parties immergées en quasi-permanence.

6. Il n'y a pas d'oxydation visible des armatures. Après un examen attentif, on ne remarque que quelques «accidents» très rares et localisés, dus à un défaut de conception ou à un défaut d'exécution. C'est le cas, par exemple, de certaines parties du pont routier sur les pertuis ou de certains ouvrages exécutés en fin de chantier (voir fig. $6,7,8$ et 9). Cela démontre, s'il en était besoin, l'impérieuse nécessité de respecter les prescriptions imposées et, notamment, le positionnement des armatures à $5 \mathrm{~cm}$ des parements.

Sur le parement inférieur de certaines poutres, on voit de nombreuses taches de rouille. Elles ne proviennent pas des armatures, mais des chutes de fil à ligature, tombées en cours de ferraillage sur le fond du coffrage et qu'il n'a pas été facile ou même possible d'enlever.

Heureusement, cela n'a pas de conséquences.

\section{3. - Déformation de la digue}

La digue est un ouvrage de structure mixte, comprenant un voile central en béton et du remblai de part et d'autre. Celui-ci est chargé en surface par des enrochements de plus en plus gros, afin d'être stables sous l'action de la houle et de former filtre pour la circulation de l'eau dans l'intérieur du massif.

On avait hésité, à la conception, entre un voile très mince en béton armé, facilement déformable sous l'action des poussées venant soit du côté mer, soit du côté bassin, et un mur épais. Finalement, l'ouvrage a été réalisé avec un mur moyen de $9 \mathrm{~m}$ d'épaisseur à la base et $2 \mathrm{~m}$ au sommet pour une hauteur moyenne de $22 \mathrm{~m}$.

Ce mur présente une galerie de pied et des puits pour pendules afin de mesurer ses déformations.

Lors de la mise en eau, la déformation maximum en tête a été de $2,9 \mathrm{~mm}$ pour une charge d'eau de $11,50 \mathrm{~m}$, le 29 mars 1966

Les remblais (supportant les passerelles piétons) se sont tassés :

- au nord: de $7 \mathrm{~cm}$ pendant la mise en eau, du 16 mars au 8 avril 1966, puis de 5 à $8 \mathrm{~cm}$, entre décembre 1966 et mars 1968.

- au sud: de $25 \mathrm{~cm}$, puis de $7 \mathrm{~cm}$ pendant les mêmes. périodes.

Depuis mars 1968 les tassements sont très faibles.

Ainsi donc, il n'y a pas eu de désordre dans la digue et chaque partie de l'ouvrage a correctement joué son rôle. 


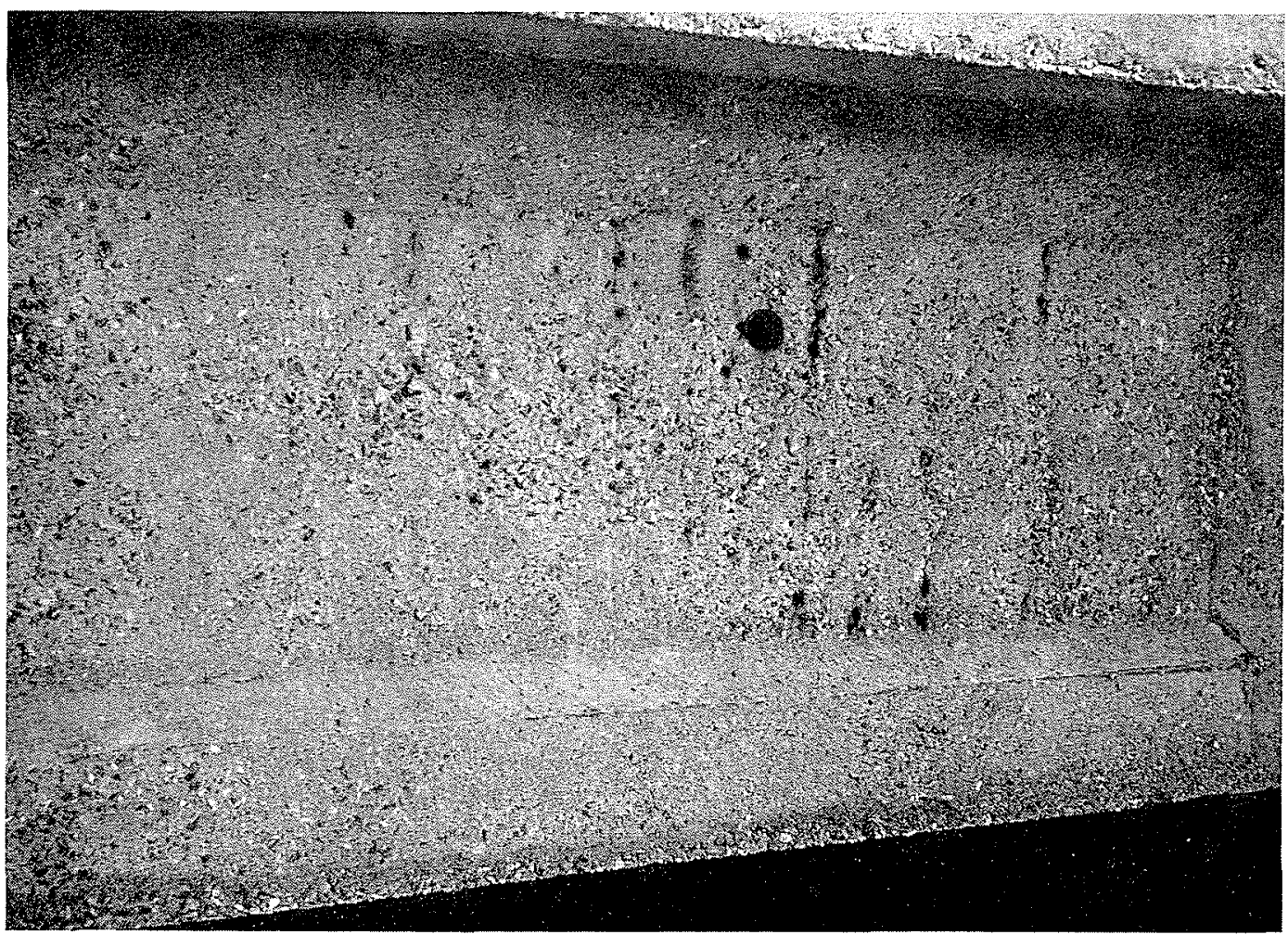

6/ Pont-route sur pertuis : poutre préfabriquée.

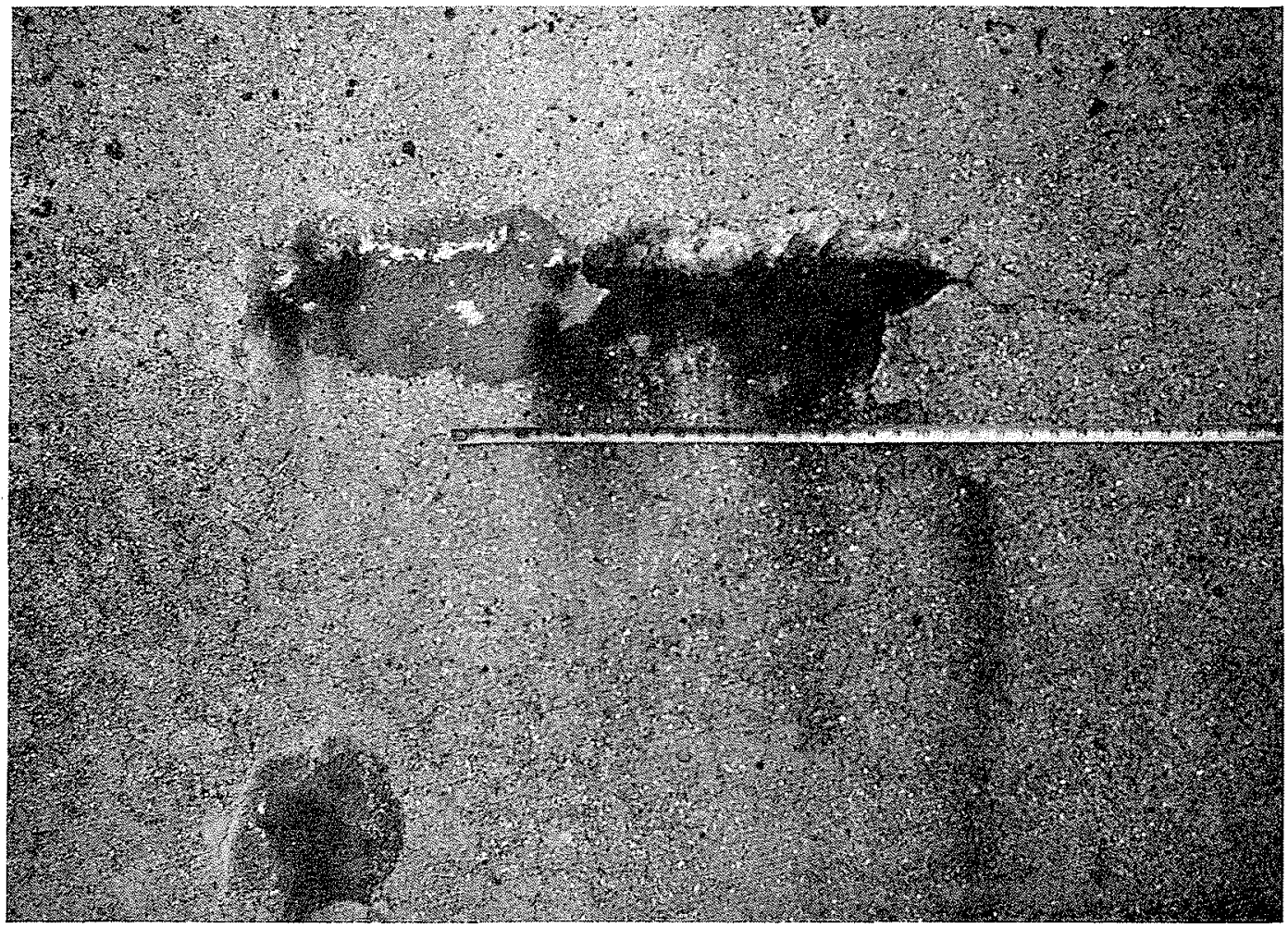

7/ Exemple d'un des rares «accidents». 


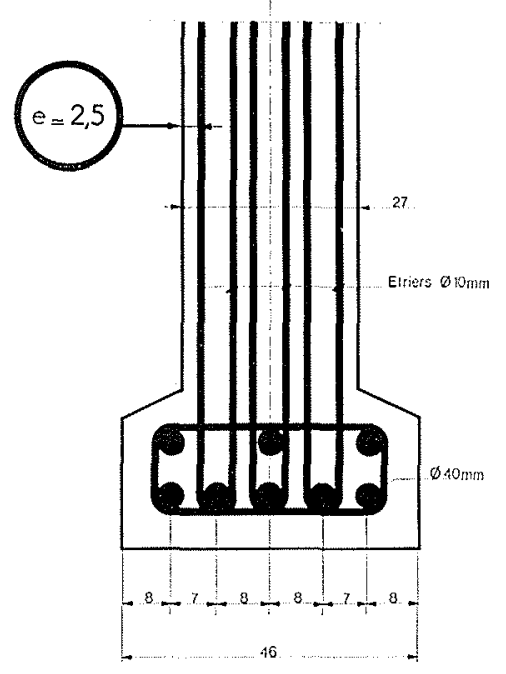

8/ Pont-route sur pertuis: poutre préfabriquée.

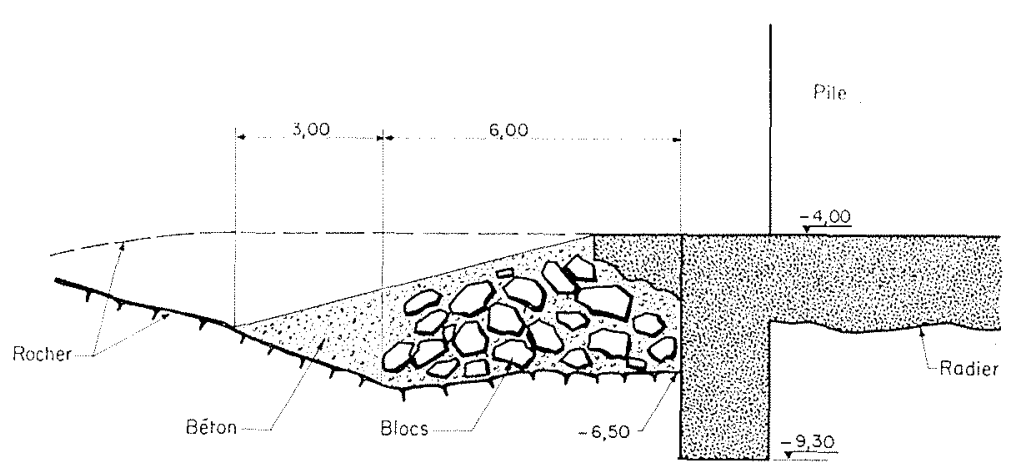

10/ Affouillement devant $P 3$, largetr $6 \mathrm{~m}$ environ.

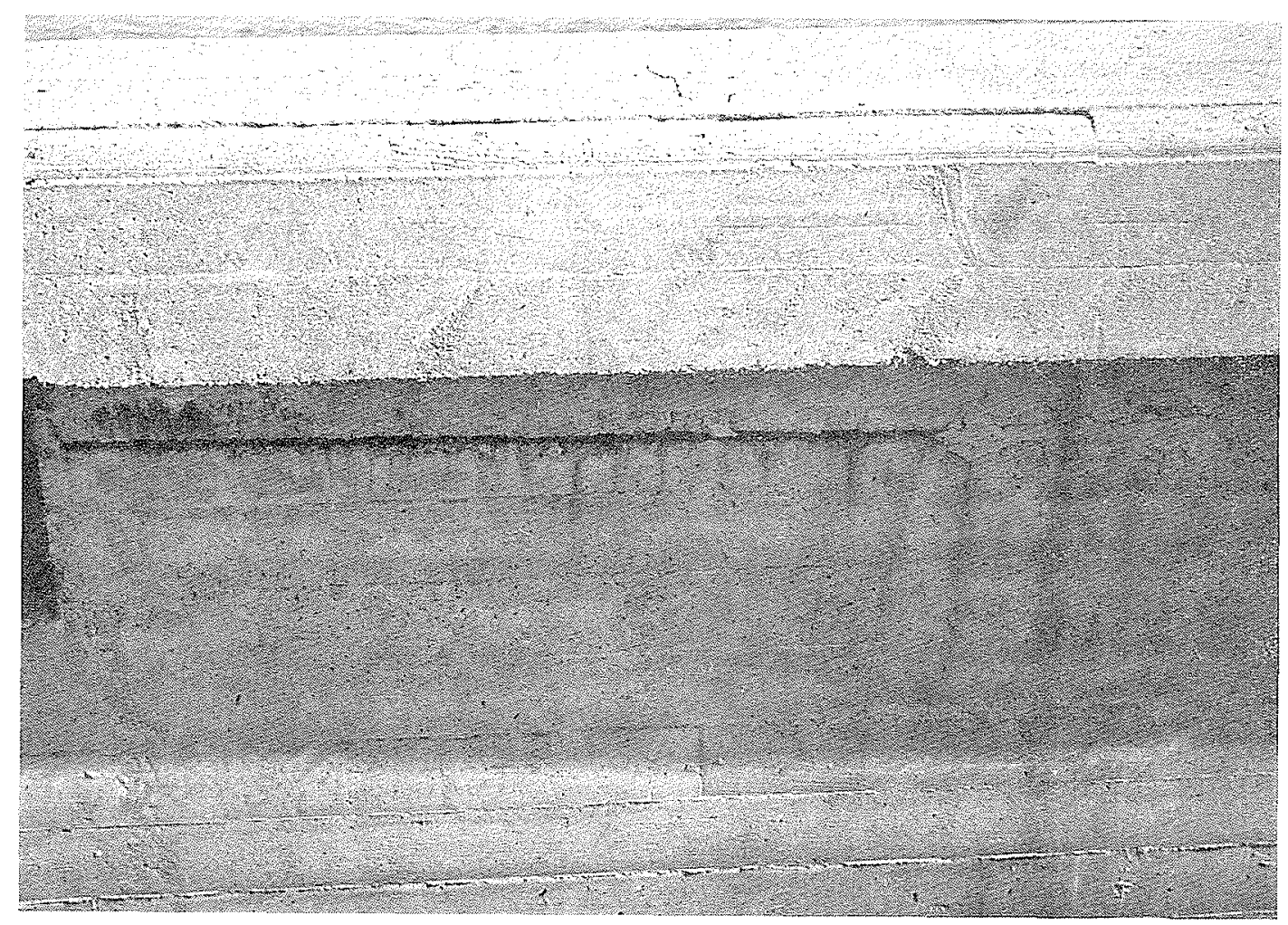

9/ Elément de passerelle pour piétons. Exemple de corrosion d'une armature mal positionnée. 


\section{4. - Affouillements}

Le rocher de fondation est une granulite feuilletée avec présence de micaschistes. Il est traversé par des filons de diabase, roche de fusion microgrenue, dure, très fragmentée, à base de feldspath et de pyroxène.

Lors de la construction du batardeau, la tenue de ce rocher, sous l'action des courants, a donné beaucoup de soucis et il y eut des périodes critiques. Pour éviter des dégradations aux ouvrages définitifs, les murs parafouilles ont été descendus profondément dans le rocher sain et l'ensemble du terrassement a été exécuté avec beaucoup de soin. Une faille au droit du G 18 avait été largement vidée et remplie de béton. Pendant ces six années, le Service de la Production n'a remarqué aucun affouillement devant les groupes et son attention a été seulement portée sur un affouillement au droit du pertuis $\mathrm{n}^{\circ} 3$, côté bassin.

Lors de l'exécution, les parafouilles avaient été descendus jusqu'à $(-10,00)$ environ (ce qui donne une épaisseur de $6 \mathrm{~m}$ de béton à partir du radier), et ils avaient été complétés côté bassin par un préradier de $2 \mathrm{~m}$ de largeur.

La figure 10 montre l'évolution de la situation reconnue par des plongeurs en mai 1964 et en mars 1970.
En 1970, E.D.F. a fait procéder par la Société Sogetram au comblement de l'excavation, en y introduisant $17 \mathrm{~m}^{3}$ de blocs et $77 \mathrm{~m}^{\prime}$ de béton injecté à la pompe.

Il s'agissait d'ailleurs d'une précaution, car l'ouvrage n'était aucunement menacé, et, pour l'avenir, il n'y a pas lieu d'être inquiet.

\section{5. - Conclusions}

Pendant cette période des six premières années d'exploitation de l'Usine de la Rance, les ouvrages de génie civil ont eu un bon comportement, et on peut penser qu'ils résisteront longtemps dans l'avenir.

Mais six ou dix années doivent normalement compter assez peu dans leur vie et un test de vingt-cinq ou cinquante ans serait plus probant.

Toutefois, leur bonne étanchéité, leur stabilité et leur bonne résistance à la corrosion témoignent, qu'en subissant régulièrement des travaux normaux d'entretien, ils doivent remplir parfaitement leur rôle et concourir à faire de l'Usine Marémotrice de la Rance une réussite de la technique française.

\section{Discussion}

Président: M. P. WYART

M. le Président remercie $M$. Duhoux de son exposé très vivant et fort applaudi. Il ouvre ensuite la discussion.

M. R. Soulas (Société Générale d'Entreprises) intervient en ces termes:

Je remercie d'abord M. Duhoux pour les éloges qu'il a bien voulu décerner à ceux qui, sous la direction de MM. Mauboussin, Reynaud, Duhoux et Mabilleau ont exécuté le génie civil de l'usine de la Rance.

Permettez-moi d'insister sur deux points évoqués dans votre très intéressant exposé et de vous poser une question.

D'abord, les matériaux constitutifs des bétons. Je voudrais rappeler que, malgré les recherches faites dans la région, nous n’avons pas pu trouver un sable tout à fait satisfaisant. Nous avons donc pris le moins mauvais disponible. Il manquait notamment d'éléments fins, ce qui a conduit à forcer sur le dosage du ciment.

Ensuite, la question des fissures. Vous avez signalé les mesures prises pour y remédier. Quant à la raison de ces fissures, je crois qu'il faut signaler les problèmes posés par le calcul des structures de l'usine par suite de la disposition de l'équipement électromécanique et des efforts engendrés à la fois par les groupes bulbe et par les charges alternées de l'eau environnante. Les calculateurs ont eu beaucoup de difficultés à reprendre ces efforts dans une structure creuse, découpée un peu comme du gruyère. Il en est résulté de nombreuses variations de section qui expliquent, je crois, que l'on n'ait pas pu éviter l'apparition de fissures.

Les mesures prises pour y remédier se sont révélées très efficaces. Notons au passage qu'dles ont coûté environ $2 \%$ du prix du génie civil de l'usine, ce qui n'est pas énorme, eu égard à l'enjeu. Encore ces mesures comportaient-elles un revêtement complet des voiles extérieurs, qui était peut-être une précaution supplémentaire, non strictement indispensable. Je crois me souvenir que l'on avait laissé au moins une travée non revêtue et j'aimerais vous demander si le béton de cette travée s'est révélé moins étanche que celui des autres, ou a subi une attaque plus grande.

M. Duhoux répond ainsi à M. SOulas :

Effectivement nous n'avons pas utilisé un sable optimum. Comme presque tous les sables de mer, il manquait d'éléments fins au-dessous de $0,2 \mathrm{~mm}$. Le dosage riche en ciment et l'utilisation d'adjuvants ont partiellement compensé ce défaut.

En ce qui concerne la fissuration, je suis tout à fait d'accord avec vous pour reconnaître qu'elle a été grandement favorisée par la forme des structures. Presque partout, elle a été d'origine thermique et était très difficilement évitable. L'emploi d'un béton moins richement dosé risquait dentrainer des décompositions chimiques et la multiplication du nombre des levées créait des reprises supplémentaires. La franche fissure de retrait thermique était donc un moindre mal.

Enfin, en ce qui concerne les revêtements d'étanchéité, je dois indiquer que les voiles extérieurs des travées de groupe n'ont reçu qu'un traitement incomplet destiné surtout à préserver le béton de l'action de l'eau de mer.

Comme vous l'avez dit, une des travées n'a pas reçu de revêtement. Son étanchéité est aussi bonne que celle des autres travées, mais il faut toutefois préciser que les fissures et reprises y ont été traitées, ce qui constitue l'essentiel pour le comportement pendant une ou plusieurs dizaines d'années.

M. Robert (Ministère du Développement industriel et scientifique) pose quelques questions au sujet du contrôle des bétons. M. DuHoux, dit-il, a souligné le soin avec lequel ont été conçus et construits les ouvrages en béton armé de la Rance. Il a indiqué qu'un contrôle très attentif de la fabrication du béton avait été assuré. Je présume que ce contrôle n'a pu que confirmer la qualité des ouvrages.

Une étude statistique des résultats d'essais a-t-elle eu lieu? Quelles en sont les condusions? Pourrait-on avoir communication de cette étude, si elle a été faite?

Enfin, les mesures de résistance sur des éprouvettes, piélevées par carottage dans la masse ont été faites. Quels sont les résultats obtenus, par comparaison avec les éprouvettes de contrôle de fabrication?

Je pose ces questions car je fais partie d'une Commission qui étudie actuellement la question de la résistance des bétons et du contrôle de leur fabrication. 
Sur la première question, M. DuHoux répond:

Dans une première phase, après la fin des travaux de l'écluse et des pertuis de vannage, nous avons fait une analyse statistique des résultats d'essais de béton de façon à en tirer des enseignements pour les bétons de l'usine. Ce document interne à E.D.F. pourrait certainement vous être communiqué.

De même, pour l'usine, nous avons établi un document important comportant une analyse de tous nos contrôles de ciment, de sable et de béton. Ces contrôles se placent dans de nombreux domaines: résistance mécanique, porosité, etc.

Ces documents pourraient certainement vous être communiqués.

Grâce à ces contrôles, nous avons mis en lumière les résultats qui prouvaient que le béton était de bonne qualité :

- d'une part, parce que la pâte de ciment est très dure et qu'elle durcira encore très longtemps;

- d'autre part, parce que la liaison du caillou avec la pâte de ciment est excellente.

Sur le problème de «l'indice de carottage », M. DuHoux s'exprime ainsi :

Pour l'exécution des carottages dans les bétons des ouvrages nous utilisions des outils diamantés importants qui donnaient un diamètre d'échantillon égal à celui des éprouvettes, soit $150 \mathrm{~mm}$.

En analysant tous les résultats d'essais de résistance obtenus sur béton frais, les points expérimentaux se groupent dans un fuseau on constate que les résultats afférents aux carottages se placent presque toujours dans la partie supérieure du fuseau. Les carottages donnent de façon systématique des résistances un peu meilleures que la moyenne des bétons frais... mais ils restent quand même dans le «fuseau».

Sur une question de M. Robert, M. DuHoux indique que le coefficient de variation (écart quadratique moyen relatif) des séries de mesures est de l'ordre de 8 à $9 \%$ à 90 jours, ce qui est remarquable si l'on observe que cette dispersion comprend celles dues la confection de l'éprouvette, au processus d'écrasement de celle-ci, au type de machine employé, etc.

Mais le contrôle ne suffit pas. Il est presque inefficace si, au départ, on n'a pas pris les bonnes options, si l'on n'a pas obligé l'entrepreneur à respecter certaines contraintes et consenti un supplément de prix pour composer un bon béton

M. LE MAY (E.D.F. Equipement) sollicite quelques renseignements sur les résultats des essais effectués sur des éprouvettes en béton armé, placées en Rance, en vue d'étudier le problème de la corrosion des armatures.

Tout près du barrage, explique $M$. Duhoux, nous avions mis 120 éprollvettes en observation sur une estacade en bois.

Ces éprouvettes étaient exécutées avec deux qualités de béton et certaines avaient été volontairement fissurées par flexion.

Les armatures étaient constituées par un rond d'acier associé à un demi-tube (dans ce profilé la corrosion est rapidement néfaste en raison de la grande valeur du rapport surface/section).

L'acier était soit de l'acier doux ordinaire, soit de l'acier galvanisé, avec ou sans protection cathodique.

L'épaisseur. du béton de recouvrement variait de 3 à $9 \mathrm{~cm}$ et les éprouvettes étaient placées à quatre niveaux différents.

Tout récemment, ces éprouvettes ont été examinées (l'analyse fera l'objet d'une publication).

On peut ainsi résumer les conclusions :

$1^{\circ}$ Le facteur le plus important de corrosion a été la fissuration. Très souvent il y a eu coupure du demi-tube dans les poutres fissurées.

$2^{\circ}$ L'épaisseur du recouvrement est très importante et la corrosion diminue quand cette épaisseur augmente. La valeur de $5 \mathrm{~cm}$ semble acceptable . 11 faut toutefois remarquer que la qualité des deux bétons était supérieure à ce qu'elle peut être dans certaines zones d'ouvrages Or, on sait que la porosité excessive du béton entraine généralement la corrosion sur toute la longueur de l'armature.

Ce facteur qualité du béton entre armature et parement est certainement aussi important que celui de l'épaisseur de recouvrement.

$3^{\circ}$ On a noté un excellent comportement des armatures galvanisées. Cola peut surprendre si on garde en mémoire les anciens préjugés techniques à ce sujet; mais cette constatation est tout à fait conforme avec les expérimentations et observations récentes, aussi bien en France qu'aux Etats-Unis.

L'adhérence était excellente, meilleure que sur l'acier nu.

$4^{\circ}$ La protection cathodique a été efficace pour la protection des armatures.

$5^{\circ}$ Le niveau le plus attaqué était la zone supéricure de marnage.

M. le Président clôt la discussion en remerciant M. Duroux et les personnes qui sont intervenues dans la discussion. 
